\title{
Jóvenes indígenas universitarios en la zona metropolitana de Guadalajara
}

\section{Indigenous Students in Guadalajara Metropolitan Area}

Isaura Matilde García Hernández*, Ana Lilia Garcia Cortez*, Juan Carlos Silas*

\section{RESUMEN}

Este texto da cuenta de la conformación de un grupo de Jóvenes Indígenas Universitarios en la Zona Metropolitana de Guadalajara (ZMG) que tuvo la iniciativa de generar espacios y acciones propios para reflexionar sobre los factores (migración, educación e identidad) que influyen en su formación personal y profesional. Un propósito adicional fue bosquejar acciones para atender colectivamente las problemáticas que atañen a los indígenas en el contexto de la ciudad. Se presenta la sistematización de sus reflexiones en el marco de tres encuentros organizados de manera autogestiva en 2014. Dentro del proceso se tuvieron cuatro resultados principales: 1) generar un clima de confianza que permita la expresión libre de ideas; 2) analizar vivencias comunes para llegar a reflexiones que difícilmente se hubieran logrado sin este diálogo; 3) fortalecer la identidad múltiple como joven indígena universitario, y 4) abrir oportunidades de discusión que favorecen la capacidad de agencia de los jóvenes indígenas universitarios como gestores en la creación de oportunidades propias y para otros jóvenes indígenas.

Palabras clave: jóvenes, indígenas, universitarios, etnicidad, ciudad

\section{ABSTRACT}

This text gives an account of the formation of a group of Indigenous University Students in the Metropolitan Area of Guadalajara (ZMG) who had the initiative to generate their own spaces and actions to reflect on the factors (migration, education and identity) that influence their personal and professional formation. An additional purpose was to outline actions to collectively address the problems that affect indigenous people in the context of the city. The systematization of their reflections is presented within the framework of three meetings organized in a self-managed way in 2014. Within the process, there were four main results: 1) Create a climate of trust that allows the free expression of ideas; 2) analyze common experiences that allow us to reach reflections that had been hardly achieved without this dialogue; 3 ) strengthen multiple identity as a young indigenous university student; and 4) open discussion opportunities that favor the agency capacity of indigenous university students as managers in the creation of their own opportunities and for other indigenous youth.

Key words: young, indigenous, university students, ethnicity, city

\section{INTRODUCCIÓN}

Si bien, la presencia indígena en la ciudad ha cobrado fuerza, es claro que sigue siendo invisibilizada, básicamente porque la ciudad es un contexto hostil hacia la diversidad cultural. Domínguez

*Universidad de Guadalajara, México; isaura_maty@hotmail.com

× Integrante del Colectivo JIU, México; garcia.ana08.ag@gmail.com.

- Instituto Tecnológico y de Estudios Superiores de Occidente (ITESO), México;

silasjc@iteso.mx. 
(2013) menciona que encontrar indígenas en la ciudad no es ni ha sido un fenómeno social nuevo, existen registros de presencia de pueblos originarios en la ciudad desde los años treinta; lo novedoso en la actualidad es que, ya no lo hacen de manera estacional o temporal, sino que desde los años noventa se ha registrado permanencia definitiva en la ciudad, por lo tanto, es un fenómeno demográfico, social y cultural que interviene en el proceso de urbanización. De la Peña (2010) menciona que muchas comunidades se vieron obligadas a integrarse a la modernización de la vida urbana, mientras que otras, por su vínculo y organización comunal, conservan su identidad. Otros tuvieron que dejar el campo para llegar a la ciudad, y a pesar de la dinámica de la vida urbana, han hecho esfuerzos para preservar la lógica de comunalidad y el vínculo con sus pueblos, por lo que manifiestan algunos elementos de sus culturas que disocian en un entorno que espera asimilación a las costumbres urbanas.

En la actualidad, las familias y los individuos jóvenes indíge200 nas, pese a compartir la experiencia de encontrarse en un contexto que no premia su apego cultural, tienen algunas diferencias importantes. Las nuevas generaciones acceden a más oportunidades educativas y tienen un mayor grado de escolarización, en específico la educación superior. Urteaga (2017) señala como ejemplo de ello la creación de las universidades interculturales (ubicadas cerca de las locaciones indígenas) pero, para los que viven en las ciudades, la única opción son las universidades convencionales. La presencia de los jóvenes indígenas en la educación superior se ha incrementado en los últimos años.

La participación de jóvenes indígenas en la educación superior no es un fenómeno nuevo, desde hace décadas ha habido presencia esporádica de muchachos provenientes de las diferentes culturas originarias en las aulas universitarias. Sin embargo, no se cuenta con un registro confiable y los datos que se tienen antes del año 2000 son más bien anecdóticos. A partir del cambio de siglo, dado el desarrollo de nuevas políticas públicas orientadas a atender a la población marginada e indígena, se hacía indispensable crear los mecanismos para el seguimiento. A raíz de esto existe información sobre la atención a población indígena que aportan las universidades participantes en iniciativas como el Programa 
de Apoyo a Estudiantes Indígenas en Instituciones de Educación Superior (PAEIIES) de la Asociación Nacional de Universidades e Instituciones de Educación Superior. Según Casillas y Santini (2009) en 2006 se atendía a 6072 estudiantes en las universidades públicas nacionales, lo que es una muy baja proporción comparada con los casi dos millones que atendía el sistema universitario público. Las universidades interculturales, cuya operación inició con el sexenio de Vicente Fox (2000-2006), han incrementado su matrícula paulatinamente, pero no dejan de ser una parte marginal del sistema educacional superior mexicano. Se hace referencia a estas iniciativas para mostrar cómo el incipiente desarrollo de la educación superior enfocada en la población indígena no ha calado hondo aún en la población y de alguna manera obliga a los jóvenes a buscar por sí mismos un lugar en las instituciones educativas y la sociedad urbana en general.

Ante este panorama, el hecho de que un grupo de jóvenes indígenas con estudios universitarios tenga la iniciativa de repensar sus trayectos y hablar desde sus propias experiencias es importante, especialmente a la luz de sus intenciones de convertirse en actores sociales atendiendo algunas de las problemáticas de los pueblos originarios en el contexto urbano.

El propósito de este texto es dar cuenta de un proceso inédito en la ciudad: la conformación de un grupo de Jóvenes Indígenas Universitarios en la Zona Metropolitana de Guadalajara (ZMG). Para ello se recurre a describir sus reflexiones, compartidas en el marco de los tres encuentros organizados por ellos y ellas, que tuvieron lugar en 2014.

La iniciativa de conformar un grupo de Jóvenes indígenas universitarios nace de la necesidad sentida por los muchachos de reflexionar acerca de sus vivencias, a partir de sus múltiples papeles sociales y las negociaciones de identidad que llevan a cabo. Se trata de 1) jóvenes, 2) indígenas, 3) universitarios, 4) migrantes en la ciudad y 5) mujeres u hombres. El grupo base se integró por tres Jóvenes indígenas universitarios residentes en Guadalajara y estudiantes de diferentes instituciones; a) mujer ñu savi, egresada de psicología del Instituto Tecnológico de Estudios Superiores y Occidente (ITESO), b) mujer ch'ol, egresada de biología de la Universidad de Guadalajara (UDG), y c) hombre wixárika, estu- 
diante de la carrera de ciencias políticas en el Instituto Tecnológico de Estudios Superiores y Occidente (ITESO). Estos tres jóvenes invitaron a otros cuatro para conformar el equipo promotor; d) mujer cho'l, alumna de estudios políticos y de gobierno en la Universidad de Guadalajara (UDG), e) mujer wixárika, estudiante de diseño en el Centro Universitario de Tonalá (CUTONALA), f) mujer ñu savi, estudiante de ciencias de la educación en ITESO y g) hombre náhuatl, egresado de la licenciatura de derecho en la Universidad Tecnológica de Guadalajara (UTEG).

Como parte de los trabajos de conformación del grupo y de manera previa a los encuentros y reflexiones que se describen en la sección de resultados, en la primera etapa el equipo base partió de un conteo/diagnóstico que implicaba saber el número de estudiantes indígenas en instituciones gubernamentales y educativas ubicadas en la ZMG. Esto se inició en octubre del 2013 y tuvo como resultado el documento interno llamado "El Informe JIU 20132014" (JIU, 2014) en el que se rescata una base de datos con los siguientes elementos: universidad, programa y cultura originaria.

El rastreo e información para encontrar a los jóvenes indígenas estudiantes en la ciudad fue difícil. Se partió de las bases de datos de tres programas de becas económicas que favorecen a estudiantes a nivel superior; Programa Becas Indígenas de la Secretaría de Desarrollo e Integración Social (SEDIS), el Programa Nacional de Becas de Estudio Superior (PRONABES) y el Programa de Becas para Educación Superior y Titulación de la Comisión Nacional para el Desarrollo de los Pueblos Indígenas (CDI), sólo dos; becas indígenas de SEDIS y PRONABES facilitaron información.

La mayoría de las instituciones no cuenta con datos sobre el origen étnico de los estudiantes. Desconocen la cantidad de estudiantes indígenas que asisten, el programa al que están inscritos y el pueblo originario al que pertenecen. Sólo dos instituciones, una pública (UDG) y una privada (ITESO) cuentan con datos de sus estudiantes indígenas y con iniciativas que buscan dar acompañamiento a los muchachos en su proceso formativo. También tienen funcionando un programa de becas o de financiamiento. Otras dos instituciones, el Centro Educativo Nuevo Cultural Social (CENCUS) y el Instituto Tecnológico de Tlajomulco (ITTJ) reconocen tener estudiantes indígenas, pero no ofrecen 
programas de acompañamiento ni becas. Las demás instituciones consultadas: Universidad Lamar, Instituto Tecnológico Superior Zapopan (ITSZ), Centro Universitario UTEG, Universidad y UNE, la Universidad Politécnica ZMG (UPZMG), la Universidad Tecnológica de Jalisco (UTJ), y Universidad Pedagógica Nacional (UPN), no tienen datos sobre sus estudiantes indígenas y no ofrecen espacios específicos para los mismos.

Una vez que se tuvo un poco de información ofrecida por las instituciones, se usaron estos datos y las redes sociales para localizar a otros jóvenes e invitarlos a conformar el colectivo. Se invitó a 60 de los 125 jóvenes estudiantes en las bases de datos. No se pudo convidar a los restantes 65 , porque sus datos telefónicos o de correo electrónico estaban incorrectos o desactualizados. De esos 60 no llegó ninguno, lo que demostró que las vías oficiales fueron inútiles para este propósito. Finalmente, asistieron 23 indígenas universitarios que vieron la invitación abierta por redes sociales, carteles y medios de comunicación.

La invitación hacía referencia a participar en tres encuentros, uno cada mes, durante abril, mayo y junio de 2014. El primer encuentro trató el tema de la identidad, el foco del segundo encuentro fue la migración a la ciudad y el tercero trató sobre la educación.

En las siguientes secciones se presenta una breve revisión de conceptos teóricos (que fueron revisados por los jóvenes), los resultados de cada uno de los tres encuentros y un análisis esquemático de los mismos, y por último una discusión empírica-conceptual que desemboca en las conclusiones y algunas recomendaciones.

\section{A MANERA DE MARCO CONCEPTUAL}

$\mathrm{El}$ análisis de la población indígena parece centrarse en contextos rurales, por estar más cerca del imaginario colectivo de la comunidad agrícola-natural, y no tanto en los indígenas urbanos que viven en las ciudades ya sea por motivos de migración e incluso por nacimiento. Esta perspectiva es comprensible pues se basa en las que tienen los estudiosos de la población indígena y de las políticas enfocadas en los pueblos originarios, fenómeno que se ha llamado indigenismo. Éste ha atravesado básicamente tres mo- 
mentos (Korsbaek, Sámano-Rentería, 2007): 1) el que antecede a las instituciones nacionales, que va desde la invasión y conquista por parte de las potencias europeas; 2) el institucionalizado, que por obvias razones abarca desde la posrevolución, se fortalece con la fundación del Instituto Nacional Indigenista y se puede decir que termina con el siglo XX, y 3) el periodo actual, llamado neoindigenismo, que toma una perspectiva más inclusiva.

En todo caso, el indígena ha sido percibido, desde cualquier etapa o perspectiva como "el otro diferente". Primero era distinto al que había llegado de otro continente, después lo fue con relación al modelo mestizo/mestizante propuesto por los gobiernos emanados de la revolución. Ahora es distinto porque no se parece al sujeto global que proponen los organismos internacionales. El "indio" es alguien diferente, cuya caracterización ha sido consistentemente elaborada por otros, que están en capacidad de denominar a los demás con base en su percepción. "El status de indio tuvo naturaleza subjetiva, puesto que dependía de características 204 percibidas, más que de atributos innatos o inmutables" (Ávila, 2010).

La población indígena en las ciudades ha sido poco estudiada, posiblemente por su capacidad de mimetizarse con los "pobres urbanos" o porque los encargados de generar el conocimiento sobre ellos no han visto el fenómeno con la suficiente intensidad. En todo caso, la experiencia de los jóvenes indígenas urbanos que han participado en la educación superior, y la reconstrucción académica de estas experiencias, parece centrarse en tres temas: 1) identidad, 2) migración y 3) educación, los que se tocan brevemente en las siguientes secciones.

\section{Conceptos sobre la identidad}

Este tema es central en la vida de los individuos y las comunidades, especialmente las urbanas y, paradójicamente, no parece tener en la vida cotidiana la discusión y repercusión que podría esperarse. La identidad no es algo que surge solo, sino que se trata de un proceso de emergencia (es decir que surge) y de afirmación, en diálogo o confrontación con otras identidades. Es entonces el resultado de un proceso de interacción social, plural que encierra 
contradicciones y frecuentemente implica relaciones desiguales (Giménez, 2009). Existen muchas formas de entender el concepto de identidad y una diversidad de disciplinas desde las que se aborda. Para Manuel Castells (1998) no existe duda de que todas las identidades son construidas, y en este sentido es importante estudiar las condiciones y elementos que influyen en esta construcción. Por otro lado, la pertenencia a un grupo o comunidad implica compartir el complejo simbólico-cultural y se relaciona con el concepto de representaciones sociales. Hamelink (1989) cuestiona el concepto de identidad cultural señalando que ese término se aplica a la cultura, no a los individuos. En este sentido, los individuos, que se nutren de las características de una determinada cultura van definiendo sus pautas de acción e interacción. Por tanto, se afirma que pertenecer a un grupo o comunidad implica compartir y aceptar el núcleo de representaciones sociales que los caracteriza y define.

De acuerdo con Giménez (2009) existen tres series de elementos que sirven como diferenciadores de la identidad de las personas: 1) la pertenencia a una pluralidad de colectivos, 2) la presencia de un conjunto de atributos idiosincráticos o relacionales y 3) una narrativa biográfica que recoge la historia de vida y la trayectoria social de la persona considerada.

En el caso concreto de los Jóvenes indígenas universitarios, la pertenencia a grupos tanto en sus comunidades de origen como en las urbanas, así como tener una serie de atributos relacionales y personales es clave. La manera de interactuar con coetáneos, compañeros y otros actores sociales desde la identidad de Jóvenes indígenas universitarios representa un reto, pues regularmente no se analizan las identidades plurales.

La juventud se reconoce de forma diferenciada en los distintos grupos sociales, de acuerdo con su momento histórico, los elementos culturales y las circunstancias particulares. Se puede decir que la juventud es "una norma construida históricamente, desarrollada socialmente e interiorizada psicológicamente" (Souto, 2007, p. 181), de esta manera es también "una categoría construida culturalmente, no se trata de una esencia y, en tal sentido, la mutabilidad de los criterios que fijan los límites y comportamientos de lo juvenil está necesariamente vinculada a los contex- 
tos sociohistóricos producidos por las relaciones de fuerza en una determinada sociedad" (Reguillo, 2003, p. 104).

La juventud, entendida como fenómeno social, impacta a la comunidad de diferentes maneras y en distintos ámbitos como son la vida política, económica y cultural del país (Saraví, 2010). Los jóvenes indígenas realizan diferentes actividades basadas en su manera de entender su participación en comunidad. Estas acciones pueden lograr impactos en su entorno y con ello fortalecer o reconstruir su identidad. Uno de los impactos de los Jóvenes indígenas universitarios es la visibilización ante el resto de la comunidad, la que se logra, inicialmente, a través de un proceso de reconocimiento y aceptación de ellos y ellas sobre su ser (ñu savi, wixárika, cho'l, nahua, ayuuk, entre otras). Tras la visibilización, se obtienen espacios de diálogo con otros, ser interlocutor en la construcción de políticas públicas o incluso espacios en medios de comunicación. Con esto, aparecen nuevas formas de ser joven y de ver a los jóvenes, lo que representa 206 un proceso desconocido en las comunidades y en las relaciones entre los mismos jóvenes y los adultos. Los Jóvenes indígenas universitarios repiensan su identidad cultural como a) jóvenes, b) indígenas, c) universitarios, d) urbanos de una manera global, es decir, como Abou (1995, p. 40) señala: "es una constelación de identificaciones particulares en instancias culturales distintas" y por ello no puede ser homogénea, pero sí homóloga, por tanto, la vivencia de invisibilidad en la ciudad, de discriminación en escuelas de nivel básico, las dinámicas migratorias y la penetración de los medios son posiblemente los factores que influyen de manera más marcada en la vida juvenil y en su estudio (Saraví, 2010). Una característica de estas nuevas juventudes es que su proceso de escolarización fungió como un espacio/momento que ayudó a cuestionar y analizar su formación personal y profesional.

\section{Conceptos sobre migración}

Se usa este término para referirse a "el cambio de residencia de una o varias personas de manera temporal o definitiva, generalmente con la intención de mejorar su situación económica, así como su desarrollo personal y familiar" (INEGI, 2010, primer párr.). 
En México, la migración del medio rural a las ciudades aumentó sustancialmente en las décadas de los cuarenta y los ochenta. Esto influido mayormente por presiones socioeconómicas o territoriales favorecidas por la oferta de empleo de las industrias (De la Peña, 2010). La ZMG tiene actualmente la mayor población indígena en Jalisco, situación que comenzó a crecer notoriamente en los años setenta. Esto es evidente usando el criterio de habla de lengua indígena, que regularmente emplea el INEGI (2012).

Esta dinámica migratoria en el país permitió cuestionar afirmaciones simples sobre los indígenas a quienes se ubicaba solamente en el espacio rural o que el indígena, al llegar a la ciudad, dejaba de serlo. Tras años de análisis es claro que la migración no necesariamente equivale a un "rompimiento con el mundo de origen" (De la Peña, 2010, p. 10) o que el fin de las culturas e identidades indígenas se determina por el acceso a la vida urbana. Ante esto, la antropología recientemente ha construido la categoría de indígenas urbanos.

La ZMG es escenario tanto de indígenas migrantes como de indígenas urbanos. Los primeros no radican completamente en la metrópoli, sino que se trasladan y viven temporalmente en virtud de algún proyecto o necesidad particular (trabajo, educación o salud). Los segundos permanecen, han nacido o vivido desde pequeños en la ciudad, probablemente con sus familias y tienen ańos (en ocasiones toda su vida) de convivencia urbana, aunque es inevitable pensar que les antecede una estrategia migratoria a nivel familiar. Las migraciones en la ZMG son heterogéneas y diversas, e implican distintas formas de vinculación entre ellas y con las zonas matrices. Algunos ejemplos notables son:

- Los wixaritari de la sierra norte de Jalisco, quienes suelen trasladarse a la ZMG para trabajar y un poco menos por motivos de salud o para estudiar.

- Los otomíes, quienes ya tienen una red amplia con familias nacidas en Guadalajara, viven de manera más o menos dispersa y se juntan para ir a visitar su lugar de origen por asuntos festivos.

- Algunos grupos ñu savi por su parte, se encuentran establecidos en unidades sociales y territoriales de hasta tres genera- 
ciones, manteniendo responsabilidades en sus comunidades de origen.

Sin embargo, existe una diversidad muy grande que no es visible a la academia o a la clase política, quizá por no cumplir con la visión comunitarista, ni folclorista, como recientemente ha llamado la atención de Domínguez (2011) al reivindicar la presencia de los zoques, originarios de Chiapas.

El movimiento entre las comunidades de origen y la ciudad, es también resultado de políticas inefectivas en la ruralidad: "El incremento de la migración del campo a las ciudades ... mostró la insuficiencia del subsistema de educación indígena para atender las demandas educativas de las poblaciones étnicamente distintivas" (Durín, 2007, p. 68). Los desplazamientos internos trajeron problemas también en la cantidad y calidad de los servicios urbanos, las posibilidades de contar con datos confiables que favorezcan la planeación y las relaciones entre los habitantes (Borja y Castells, 2000). Es necesario reconocer la diversidad de pueblos originarios presentes en la ciudad, y la forma de asentarse también son distintivos de acuerdo con cada grupo y que viven y conviven de modos diferentes al tiempo que se relacionan con la ciudad de maneras desconocidas.

\section{EDUCACIÓN Y ESTUDIANTE}

Después de la Revolución mexicana se planteó que la "formación de la nación ... exigía la aculturación de toda la sociedad" (De la peña, 2005, p. 19). Este proyecto nacionalista se consolidó con Lázaro Cárdenas, y en cuanto a la población indígena, se impulsó con la creación del Instituto Nacional Indigenista (INI) en 1948, llamado proyecto indigenista, en el que la educación escolarizada fue uno de los medios más poderosos para incorporar a los indígenas al marco de este proyecto de nación.

La primera estrategia fue reclutar "jóvenes indígenas que destacaban por sus cualidades intelectuales y actitud de servicio, para entrenarlos como maestros y promotores bilingües" (De la Peña, 2005, p. 19). Algunos recibieron "becas estatales y apoyos privados para seguir sus estudios superiores en universidades y escuelas 
normales, sin perder contactos con la comunidad de origen" (De la Peña, 2005, p. 20). La apuesta nacional recaía en las comunidades indígenas y en el papel que jugarían los jóvenes indígenas escolarizados como catalizadores del proyecto nacional.

Las poblaciones indígenas en Latinoamérica, dada su diversidad, se constituían como un "problema" para la consolidación de los Estados nacionales. La idea base era que se asimilaran a la cultura mestiza predominante. Ante estas presiones, los pueblos indígenas se erigieron como actores de un movimiento social para la pluralización de las sociedades y demandaron una educación escolar acorde con sus especificidades culturales (Durín, 2007). Este tema es fundamental en los estudios sobre el indigenismo en México; sin embargo, en este texto se toca brevemente, pues no va al centro de la vivencia de los jóvenes indígenas urbanos.

El esfuerzo de ofrecer una educación intercultural en todos los niveles llegó con el cambio de siglo, ahora a través de las universidades interculturales, que presentan retos importantes. Schmelkes (2011) identificó tres: 1) la falta de recursos que afecta el desarrollo de éstos, 2) las condiciones de los alumnos y la fragilidad de dicha demanda, así como 3) los antecedentes académicos de los alumnos que llegan a ellas.

\section{El contexto. La Zona Metropolitana de Guadalajara y los Indígenas}

En la ZMG la población indígena va en aumento y, aunque no es una proporción muy alta, se comienza a notar paulatinamente. De acuerdo con el INEGI (2015), en 2015 la población indígena en Jalisco era de $11.1 \%$ y se concentraba en los municipios de Zapopan y Guadalajara, en algunos otros con historia de pueblos originarios, pero con una cantidad de habitantes muy menor, como los que integran la zona Wixárika en la colindancia de Jalisco, Nayarit, Zacatecas y Durango, o la zona Nahua en el Sur del estado.

Como se ha señalado, el reconocimiento y efectivo conteo de la población indígena es difícil dada la movilidad de los habitantes. Por otro lado, Guadalajara, capital cultural, social, económi- 
ca y política del occidente de México, con costumbres criollas y lógica comercial, suele ignorar el aporte indígena.

[Guadalajara] se destaca por ser una ciudad de fuertes rasgos europeos. Tanto en el espacio urbano como la cultura local de Guadalajara se identifican con el legado español, francés, alemán y estadounidense instruido por las diferentes olas de inmigración que experimentó esta ciudad durante los últimos cinco siglos. Sin embargo, la celebración del legado europeo oscurece la presencia e influencia de otras etnias que han ayudado a construir las tradiciones que hoy identifican y sustentan a esta ciudad; esto incluye, pero no se limita a poblaciones indígenas y de origen africano (Negrín da Silva, 2010, p. 55).

Durín (2007), menciona que este fenómeno que llama negación del derecho a la ciudad, es una situación heredada y reproducida desde la conquista. En este sentido, sabemos que Guadalajara fue, desde su fundación, una ciudad en oposición al 210 mundo indígena.

Si bien los pueblos mesoamericanos vivieron en ciudades, con la conquista, las ciudades fueron asiento del poder y, por consiguiente, de los espańoles, criollos, mestizos e indios urbanos ocupados en la servidumbre y el servicio doméstico, como artesanos, abasteciendo productos de consumo. Con ello se les redujo a colonizados e indios: se les negó el derecho a la ciudad y se les fijó en el mundo rural (Castellanos, 2003, pp. 84-86). Así, se les prohibió radicar en el interior de la ciudad y se asentaron en barrios situados fuera de la traza urbana española. De esta manera, la dicotomía rural/urbano se incorporó como atributo para identificar a los españoles y a los indios, y luego para distinguir a los mestizos de los indios, siendo las ciudades el asiento y espacio colonizador (Durin, 2007, pp. 68-69).

La visión de la ciudad se cuela a los espacios educativos, pues éstos ejercen más funciones que sólo promover conocimiento. Desde modelar "las actitudes y conductas consideradas pertinentes y valiosas, y éstas son asociadas al mundo 'moderno' y 'occidental”" (De la Peña, 2010, p. 16), hasta el de "internaliza(r) un modelo de sociedad que regula las normas y valores de las personas" (Durín, 2007). La ciudad internalizada por millones 
de habitantes es un factor poderoso en la constitución de las relaciones entre ciudadanos establecidos, grupos de ciudadanos y migrantes recientes.

Es en este contexto que surge el grupo Jóvenes indígenas universitarios, integrado por muchachos de pueblos originarios que se congregan para compartir vivencias, vislumbrar soluciones y generar sinergias entre ellos y con quienes les rodean. Para ello, los Jóvenes indígenas universitarios desarrollaron tres reuniones de reflexión que requirieron una estrategia para compartir y analizar que fuera flexible y conducente a sus necesidades. En el siguiente apartado se da cuenta de ello.

\section{MetodologíA}

En este apartado se describen muy brevemente, por un lado, las decisiones metodológicas desde lo conceptual y por el otro, desde lo empírico. En el plano conceptual, se decidió conducir un proceso inspirado en la Investigación-Acción Participativa (IAP) ya que se planteó desde el comienzo una manera de investigar con y para el grupo de jóvenes, en función de ir constituyéndose como una comunidad a través de discutir temas comunes como Jóvenes indígenas universitarios y de generar procesos de transformación reflexiva.

En palabras de Rahman y Fals Borda (1989, p. 213), la IAP, además de enfocarse en una búsqueda rigurosa de conocimientos, "es un proceso abierto de vida y de trabajo, una vivencia, una progresiva evolución hacia una transformación total y estructural de la sociedad y de la cultura con objetivos sucesivos y parcialmente coincidentes". De acuerdo con Emiro (2005), en el trabajo realizado en investigación-acción, se pueden resaltar varios rasgos distintivos, entre ellos: 1) que busca profundizar en la comprensión del problema, evitando en lo posible las posturas y las definiciones previas; 2) explica "lo que sucede" sobre la situación y sus actores, relacionándolo con su contexto; 3) parte inductivamente de una narración y no de una teoría, por ello los elementos del contexto se integran a las visiones de los actores; 4) los resultados son una interpretación más que una explicación dura; 5) se construye en la subjetividad y cómo ésta se expresa en 
el lenguaje de los participantes, y 6) implica un proceso de autorreflexión sobre sí mismos, los demás y la situación.

Ahora bien, desde la parte empírica, se debe señalar que se decidió trabajar en dos líneas: 1) visibilizar a los estudiantes indígenas en los centros universitarios y 2) realizar encuentros para reflexionar, de manera crítica y autocrítica, sobre los elementos que influyen en la formación personal y profesional. En este texto se aborda únicamente la segunda línea y se reporta la dinámica de los tres encuentros que le dieron forma.

En este punto de la descripción es fundamental señalar que dos de los autores de este texto participaron en todo el proceso, desde la organización, dinamización, la sistematización y análisis. En este sentido, en congruencia con el espíritu de la IAP $y$, con la finalidad de dar cuenta minuciosa del proceso, muchas de las ideas que se plasman en esta sección y la de resultados parten de lo recuperado en el "trabajo de campo" de una manera participativa. Esto no significa que sea un proceso sin rigor, al 212 contrario, el proceso de sistematización:

Es aquella interpretación crítica de una o varias experiencias que, a partir de su ordenamiento y reconstrucción, descubre o explicita la lógica del proceso vivido, los factores que han intervenido en dicho proceso, cómo se han relacionado entre sí, y por qué lo han hecho de ese modo (Acosta, 2005, p. 7).

En este sentido, se puede decir que es un proceso en el que se recupera la experiencia que se vivió en un contexto durante distintos eventos. Las personas encargadas de la recuperación, sistematización y análisis de testimonios, dos de los autores de este texto, son parte de los Jóvenes indígenas universitarios. Es, entonces, importante señalar que la obtención de información de los encuentros realizados en la ZMG en 2014, en abril (identidad), mayo (migración) y junio (educación), se planteó desde los Jóvenes indígenas universitarios para Jóvenes indígenas universitarios; la dinámica desarrollada, se fundamenta en una relación de horizontalidad, donde ninguna experiencia es más valiosa que otras.

Las actividades se dieron en tres momentos y giraron en torno a: 1) compartir vivencias, experiencias y expresar comentarios de 
los jóvenes con referencia a los ejes temáticos correspondientes; 2) abordar el tema con fundamentos teóricos y una presentación (Power Point), y 3) finalizar con comentarios y reflexiones colectivas de lo vivido en cada encuentro. Para recuperar dichos eventos, se hicieron grabaciones de audio y fotografías. Lo más importante son las relatorías, pues en cada una se recupera toda la experiencia, el diálogo y la presentación de cada uno de los encuentros.

Para el análisis de la información se utilizaron los programas MAXQDA y atlas.ti, ambos software que analizan datos cualitativos, ayudaron a codificar, categorizar y hacer familias de códigos. El software permitió generar reportes de códigos con citas textuales para facilitar el proceso de análisis a través de los esquemas generados con los conceptos y la relación de los conceptos comentados por los Jóvenes indígenas universitarios.

\section{RESULTADOS}

Los datos que aquí se presentan surgen del trabajo de campo y del documento interno llamado: Informe JIU 2013-2014 (JIU, 2014). Se presentan los aprendizajes colectivos que se lograron de manera transversal y que surgieron a partir de los encuentros temáticos de: abril (identidad), mayo (migración) y junio (educación). Cabe señalar que este análisis transversal aportó información rica y puntual. En el encuentro se compartieron reflexiones y experiencias de la situación indígena en la ciudad, especialmente en los temas que influyen en la vida de los Jóvenes indígenas universitarios de la ZMG. Los jóvenes expusieron ideas personales para llegar a una idea colectiva sobre los temas. No se planeó como una discusión teórica al estilo de una cátedra académica, sino que el interés fue generar un espacio de sintonía, de compartir aprendizajes y reflexiones, pues, como se dijo, el encuentro promovió relaciones horizontales y de igualdad, en que nadie sabe más que el otro.

En estos tres encuentros se siguió la misma estructura lógica, que fue: 1) ahondar en la experiencia del joven, para que él/ella comparta y reflexione sobre el tema a partir de sus vivencias, y 2) confrontar y profundizar en común esas reflexiones usando 
los ejes temáticos acordados para cada encuentro, para 3) hacer un cierre con las conclusiones generales. En este sentido se iniciaba dialogando desde la experiencia individual para pasar a la colectiva, sin excluir el uso de conceptos y, finalmente, lograr una producción común de integración.

En este punto, antes de presentar los resultados, es preciso retomar dos ideas: 1) dos de los autores del texto fueron participantes activos en el proceso y 2) la esencia de la IAP consiste en no disociar la investigación de la acción y, en este sentido, será notorio que algunos párrafos descriptivos o analíticos en los resultados y conclusiones llevarán implícita la voz de los Jóvenes indígenas universitarios como sujetos participantes en la experiencia y como autores del texto. A continuación, se presentan estos resultados.

\section{Lugar de encuentro de los Jóvenes indígenas universitarios (espacio)}

El encuentro tuvo relevancia desde el comienzo, pues cada joven tuvo la oportunidad de expresarse en libertad, en común y de manera enfocada. En el espacio de encuentro, los participantes mencionan sentirse en confianza al momento de compartir. Mencionan que el encuentro es un logro importante, ya que no se presentaba un espacio de ese tipo, es decir "de" Jóvenes indígenas universitarios "para" Jóvenes indígenas universitarios, expresaban que ya hacía falta un lugar similar donde pudieran compartir entre ellos, además que invitara a los participantes a reflexionar e incidir en temas relacionados a su cultura e identidad, tomando en cuenta la migración y la educación. Si bien el espacio del encuentro invita a motivarse sobre las posibilidades de trabajar desde diferentes ámbitos del tema indígena que ellos en algún momento ya han pensado.

No ha habido un espacio como éste, de jóvenes indígenas de diferentes culturas y este espacio va para este rumbo, ¿̨no? Podemos hacer una organización, podemos hablar sobre el tema de las becas, de los apoyos, hay leyes que hablan de los migrantes indígenas en Guadalajara, cómo acceder a ese derecho, cómo acceder a ese servicio, pero se necesita ir 
organizándonos, formar una organización civil o una organización donde nosotros podamos intervenir en estos casos, ¿no? (Joven participante del 2 do encuentro, mayo de 2014).

Creo [que] valoramos el espacio, es muy importante porque en una ciudad como Guadalajara sí se quiere un espacio, donde es difícil platicar, pero sería muy valioso, por las características de la ciudad, contrastar lugares, donde es centro político, económico y cultural, pues vale mucho la pena (Joven participante del primer encuentro, abril de 2014).

Por otra parte, el encuentro permitió reconocer la existencia de otros Jóvenes indígenas universitarios, es decir, un espacio interétnico, donde varios jóvenes indígenas de varias culturas se encuentran con su condición de migrantes, de estar en la universidad y ser mixteco, wixárika o cho'l, compartiendo los temas parecidos en un solo lugar y momento. Esto ayuda a fortalecer la identidad y amplía la visión crítica y autocrítica, abriendo una posibilidad en articular redes entre Jóvenes indígenas universitarios.

Ahora que nos conocemos y que no sabíamos dónde estamos, y acercándonos nos damos cuenta de a quién tienes enfrente, si coincides con él, qué piensa, porque si estamos con las personas o las vemos en la calle, las vemos pero, pues, nunca platicamos por el miedo de que "hay que querrá de mí”, pero, pues, igual te acercas y con lo poquito del tiempo en esta dinámica te das cuenta de lo que piensan, de sus ideales, qué los mueve; como ahí en el papelito decía "sus metas" y aunque no parezca, pues ahí están tratando de lograr algo, de salir adelante, de ayudar, de conocernos. (Joven participante del primer encuentro, abril de 2014).

Y qué bueno que se dan estos espacios porque superamos las diferencias que hay entre nosotros, somos capaces de aceptarnos, somos capaces de reconocer que estamos padeciendo lo mismo, entonces, unimos esfuerzos para salir adelante ¿̇no? (Joven participantes del segundo encuentro, mayo de 2014).

Conocen la diversidad étnica, por su experiencia vivencial, al encontrarse en lugares en común, tener referentes sobre el tema indígena o que simplemente se conocen porque se ven, el encuentro permitió compartir entre ellos las diferencias y similitudes. Se 
valoró el encuentro cuando los jóvenes se dieron cuenta de que en ese lugar podrían compartir con otros Jóvenes indígenas universitarios. Aprovecharon el espacio de encuentro para expresar todo lo que quisieran sobre el tema, mencionaron que era momento de cuestionar, analizar y reflexionar, primero, desde ellos mismos como Jóvenes indígenas universitarios para luego actuar en otro espacio. Sobre esto, un joven lo expresa de la siguiente manera: "Reflexionemos aquí, para reflexionar afuera" (Joven participante del primer encuentro, abril de 2014).

Un señalamiento común fue la necesidad de aprovechar los momentos de encuentro para compartir todas aquellas reflexiones que a nivel individual les han surgido en algún momento. En el espacio de encuentro se puede compartir con otros Jóvenes indígenas universitarios que bien pudieron identificar similitudes que los hacen ser diversos y también de reconocer y superar las diferencias entre ellos, con la finalidad de que, en adelante, pudiesen empezar a reflexionar más allá de las experiencias perso216 nales, es decir, orientar las acciones o situaciones hacia afuera. Al mismo tiempo, facilitó compartir inquietudes sobre realizar acciones para reivindicar la identidad étnica junto con su profesión.

\section{Lugar para compartir las inquietudes individuales}

Cada Joven indígena universitario convivió y conoció a otros iguales a ellos para saber de sus estudios, trabajos, reflexiones, críticas y autocrítica sobre los tres temas propuestos. En cuando a la identidad, reconocieron sus tradiciones y su cultura, sin embargo, se percatan que algunos elementos se van perdiendo o que se han perdido a lo largo del tiempo en sus personas y comunidades. Por otra parte, al reconocer y aceptar su origen e identidad indígena empiezan a pensar en los posibles compromisos y propuestas para atender problemáticas identificadas en sus vivencias particulares.

Entonces, yo digo, a mí sí me gustaría que sigan estos espacios para el día de mañana, sabes qué, pues hay que ir o hay que ir a hablar para que ellos vean que sí estamos los indígenas presentes y que podemos llegar a 
un acuerdo y trabajar, empoderarnos, trabajar todos juntos (Joven participante del primer encuentro, abril de 2014).

Tal como ya hay comentarios de otros compañeros, reforzar mucho nuestra formación académica, formación social, también ir como captando más ideas, más formas de pensar de otras personas, donde también podamos compartir con los demás, ir formando una organización, un colectivo, un proyecto de nosotros, no de uno mismo, más bien de... estamos ya formando un grupo y esto que se fortalezca más, porque entre que nosotros sigamos haciendo estas reuniones, se va fortaleciendo más (Joven participante del tercer encuentro, junio de 2014).

El Joven indígena universitario se refiere a varias situaciones y experiencias de vivir en la ciudad, se enfrentaron a retos que no esperaban, el hecho de encontrarse con otros jóvenes en este espacio les genera una nueva forma de reflexionar, ya que enriquece su visión a través de compartir sus vivencias. Estas experiencias los motivan a reconocer su perfil personal y profesional para pensar y generar acciones.

Creo yo, ahora nosotros, los que estamos aquí, sí somos del pueblo nativo, si queremos conservar, no sé, un tiempo más nuestras identidades que cada quien ejercemos, creo que tenemos que tener una reflexión muy crítica para poder nosotros empezar realmente [a pensar] qué es lo que queremos ser en un futuro, para ahí aterrizar ahora sí, a dónde queremos llegar (Joven participante del 1er encuentro, abril de 2014).

Los jóvenes indígenas reflexionaron a partir de sus experiencias, cada uno sabe que tiene mucho por hacer desde sus intereses para empezar a generar cambios e incidir en sus comunidades, en específico, para así tener impacto en la sociedad en general.

Entonces, creo que aquí la propuesta es que nosotros seamos los verdaderos promotores, que nosotros usemos nuestros espacios ... (Joven participante del primer encuentro, abril de 2014).

Lo más importante para los Jóvenes indígenas universitarios es valorar, conocer y fortalecer la identidad para tomar impulso 
anímico y seguir participando, ya sea en lo individual o en colectivo y buscar tener impacto en sus comunidades y la sociedad en general.

\section{Lugar de reflexiones colectivas}

El encuentro, aparte de generar reflexiones individuales, llevó a generar reflexiones colectivas que se reflejan a partir de la confianza y apertura que el espacio les generó. Vivieron momentos de profunda reflexión, crítica y autocrítica. Se percibieron similitudes en las experiencias y vivencias particulares de estos jóvenes y sus procesos, contribuyendo a cuestionar lo que han hecho y lo que les falta por hacer de acuerdo con sus inquietudes. Los jóvenes compartieron pensamientos y vivencias personales que se reflejan en lo colectivo, dimensionando y relacionando hacia las instituciones, con sus pares, con sus pueblos y el entorno.

Los dos temas que generaron mayor reflexión colectiva entre

218 los Jóvenes indígenas universitarios fueron: identidad y educación. Estos temas contribuyeron a recordar las implicaciones que representa la realidad de la que forman parte. Después de realizar una recuperación de su trayectoria escolar, se alcanzó a identificar cuáles son los procesos de cuestionamiento, negación, aceptación y toma de postura identitaria. Mencionaron que la educación escolarizada es un factor que determina el proceso de aprendizaje e identidad del Joven indígena universitario. En la institución escolar adquieren conocimientos de distintas disciplinas; sin embargo, no es un lugar para discutir y analizar el conocimiento indígena, de estos diálogos surgieron propuestas de mejora.

Pensamos que la educación debería tener un sistema educativo que tome en cuenta las tradiciones y costumbres de lo que son las comunidades indígenas y que formen con métodos educativos basados en las necesidades de los alumnos y lograr así un aprendizaje significativo. [Que también cuente con] el compromiso de los profesores, y de los padres de familia, y autoridades educativas, que es la tarea de todos, y así que genere una educación que realmente se espera en la comunidad. Como conclusión, que la educación está en deuda con las comunidades en cuanto a participación, en los contenidos básicos regionales y en proyectos educativos 
para comunidades indígenas, que no están tomadas en cuenta (Joven participante del tercer encuentro, junio de 2014).

Se hicieron reflexiones sobre el sistema educativo, que se identificó como homogéneo y homogeneizante a partir de su metodología y el idioma de enseñanza. Compartieron que esto complicó su incorporación a las clases y les implicó adaptarse a la forma de aprender y trabajar de los compañeros no indígenas.

Por otra parte, se señaló con insistencia que en su trayectoria escolar se enfrentaron a la discriminación por vestir de manera diferente, por hablar otro idioma o simplemente por tener otras tradiciones. Los Jóvenes indígenas universitarios atribuyen esta vivencia de discriminación al desconocimiento de la gente sobre los significados que tienen para los pueblos originarios sus tradiciones.

En cuanto a reforzar o no esa identidad, influye su trayectoria escolar, las condiciones familiares, el contexto al que pertenecen y la sociedad en general. La construcción del proceso identitario de los Jóvenes indígenas universitarios se caracteriza por dos fases: 1) la exclusión que los hace sentir menos que los demás, con su consecuente negación, debido a los fuertes prejuicios hacia su identidad étnica, y 2) la asimilación, en la que empiezan a percibirse y aceptarse, debido a que se involucran en temas indígenas y la aceptación de la identidad, donde la comprensión de la situación los lleva a ser más reflexivos y a tener mayor compromiso en su trabajo en organizaciones, participación en la comunidad y la vida en la ciudad.

\section{Exclusión y negación}

Como resultado de las ideas anteriores, los Jóvenes indígenas universitarios señalaron que el hecho de que sus compañeros desconocieran sus tradiciones y cosmogonía influía en un desprecio hacia ellas.

De los compañeros, no te relacionabas bien con [ellos] por lo mismo o por la referencia que hacían hacia uno por ser indígena. O por ejemplo aquí se compartía, que un compañero dijo que cuando entró a la primaria 
hicieron un volado para ver quién se quedaba con él (Joven participante del segundo encuentro, mayo de 2014).

Existen experiencias que marcan la vida del joven indígena, que le llevan a negarse como tal, los más comunes son los rasgos físicos, vestimenta, lengua y tradiciones. En la reflexión del Joven indígena universitario:

Yo veo que siempre es eso, la mayoría comentamos que te ven diferente, a veces la mayoría venimos más chaparritos y te dicen “¡eres indígena?” Y uno a veces como que se siente, ¿por qué o qué soy?, o te dicen "es que no hablas español", y poco a poco nos vamos acoplando. $\mathrm{O}$ a veces vamos perdiendo nuestra identidad, nos identificamos más de ciudad que en reconocer nuestra propia cultura (Joven participante del segundo encuentro, mayo de 2014).

Un elemento común en sus relatos es que perciben claramen220 te que lo tradicional y originario se está olvidando y se pierde el interés de rescatarlo, debido a que no se están generando espacios en los que se fortalezca la identidad. El Joven indígena universitario reflexiona sobre el tema de la pérdida de la cultura y, al tiempo que se lamenta por el estado de las cosas, empieza a valorarse y señala la intención de desarrollar proyectos que tienen relación con la lengua, la cultura y la identidad indígenas.

La cultura, se está perdiendo, nuestra lengua, idioma, el idioma indígena se está perdiendo en todas partes. La vestimenta, también las ceremonias se están perdiendo, ¿por qué? Porque la generación [en la] que estamos, que están siendo... los nińos pequeños ya no hablan la lengua, los jóvenes ya no quieren hablar la lengua, ellos ya están viendo otras ideas, llevan... nosotros... muchos chavos que terminan la primaria, vienen a la ciudad, ven la nueva cultura, llegan a la comunidad y llevan la música diferente, llevan la vestimenta diferente, llevan costumbres diferentes como... llevar cigarro, fumar, tomar y todo eso y están llevando ahí a la comunidad, eso es lo que cambia (Joven participante del primer encuentro, mayo de 2014). 
El Joven indígena universitario reconoce las experiencias y vivencias relacionadas con la discriminación, porque las vivieron (o las viven); sin embargo, sabe que está en su postura y decisión dejar de percibirlas, e incluso, alienta a trabajar más en la identidad de cada uno.

Nos falta muchísimo [para] realmente sentir esa pertenencia, son muy pocos los que se han atrevido ante instancias gubernamentales a decir "sabes qué, respétenos, ésta es nuestra lengua, ésta es nuestra cultura, no queremos estos factores externos que vienen a dañar nuestra unidad". Porque realmente van dańando la unidad, nuestros pueblos están perdiendo bastante... (Joven participante del tercer encuentro, junio de 2014).

\section{Asimilación y aceptación}

Una vez que son conscientes de sus problemáticas, reconocen que al aceptarse o reconocerse como joven indígena puede fortalecer su identidad, despertando mayor interés por sus tradiciones y su comunidad. Dentro de este reconocimiento JIU, surgen procesos de reflexión crítica y analítica; es decir, manifiestan reconocer y practicar su cultura, identifican algunas características de su identidad, e incluso manifiestan participar en actividades y en sistemas de cargos (organización comunitaria); hacer artesanías; hablar su lengua materna; portar la vestimenta, no vista de manera folclórica, sino que ya son conscientes de que la identidad va más allá de lo evidente.

Cuando uno acepta su identidad, sí es posible ser indígena y vivir en la ciudad ... Yo pienso que sí, porque eso está en nosotros mismos, si queremos avergonzarnos, obviamente lo vamos a perder. Pero si estamos conscientes de dónde venimos y dónde estamos y hacia dónde queremos ir, siento que sí es posible vivir en la ciudad, un indígena, manteniendo su cultura presente, viva, en su interior e incluso compartir con la gente (Joven participante del primer encuentro, abril de 2014).

Finalmente, a través de sus experiencias, mencionan que hay mucho por hacer para generar cambios en la sociedad actual, pero lo más importante es valorar, conocer y fortalecer la identidad 
para, de ahí en adelante, seguir participando, ya sea en lo individual o en colectivo, e incluso, generar otros encuentros, talleres, convivencia, participación, etcétera.

Yo creo que hay que cambiar esa idea, cambiar esa forma de pensar de la gente. Y nosotros, también nosotros, que nosotros pensemos así, que somos experimentos, que cambiemos esa creencia. Vuelvo a retomar eso, si nos vamos a poner algo que nos identifique, como para demostrarnos, no como para sentirnos vulnerables, eso sería una educación para las demás personas que están fuera (Joven participante del tercer encuentro, junio de 2014).

El Joven indígena universitario valora las oportunidades que ha tenido, sabe que es importante no sentirse inferior por ser indígena, sino saber, compartir y demostrar lo que son. Ellos reconocen su capacidad de romper prejuicios, de realizar proyectos $\mathrm{u}$ organizaciones, de entender la historicidad del tema indígena, de valorar su cultura, e incluso visibilizar el tema indígena en la ciudad. Como resultado de todas las reflexiones que compartieron de sus diferentes experiencias y vivencias en los encuentros, generaron compromisos y propuestas que son importantes porque están pensadas para las comunidades. Desde 2017 hasta la actualidad, al equipo base y promotor se suman otros integrantes, amplían su equipo a Jóvenes indígenas universitarios, ahora como urbanos han realizado tres actividades muy notorias: 1) Gestionar un espacio en la radio pública de Jalisco. El programa se llama Raíces al Aire, que ya tiene diez meses se transmisión. 2) Desarrollar talleres de difusión cultural indígena y sensibilización a la población mestiza en la ciudad. Éstos se han impartido cuatro veces en un año. 3) Desarrollar encuentros académicos en instituciones de educación superior en los que se muestran los rasgos culturales de los participantes y se expone la manera en que los Jóvenes indígenas universitarios de Guadalajara se han organizado. A la fecha se han llevado a cabo en instituciones privadas de Guadalajara y la Ciudad de México. 


\section{A MANERA DE CONCLUSIONES}

Tras hacer un recuento de las reflexiones de los Jóvenes indígenas universitarios acerca de los factores que influyen en su formación personal y profesional se puede ver que la puesta en común durante los encuentros permitió varios procesos: 1) Encontrar aceptación, un ambiente de escucha e identificación. Esto se hace evidente en la creación de un clima de confianza que permite la expresión libre de ideas. 2) Intercambiar vivencias comunes y analizarlas permitió llegar a reflexiones que difícilmente se hubieran logrado sin un diálogo enfocado y propositivo. 3) Fortalecer la identidad como Jóvenes indígenas universitarios y al mismo tiempo detonar reflexiones en torno a su valor, elemento fundamental del ser humano. 4) Abrir oportunidades de discusión favorece la capacidad de agencia de los Jóvenes indígenas universitarios quienes se asumen como posibles gestores en la creación de oportunidades propias y para otros.

Este proceso, que fue descrito contundentemente como positivo, plantea nuevos retos en la visibilización de los Jóvenes indígenas universitarios y en el acercamiento cultural con la población mestiza. Dentro de las reflexiones se señaló que el proceso migratorio es un fenómeno inevitable para muchas familias de zonas rurales e indígenas. Las dinámicas socioeconómicas que les han llevado a establecerse en la ciudad van a continuar y que traer consigo su idiosincrasia también seguirá. La dinámica relacional entre los jóvenes indígenas urbanos (sean universitarios o no) y la población en general, requiere que se dé en un marco de mayor conocimiento y respeto por parte de todos los involucrados. En este sentido, la pertenencia a un colectivo creado intencionalmente con la finalidad de reconocerse como Jóvenes indígenas universitarios y de reflexionar la trascendencia de las vivencias comunes, se presenta como elemento actuante en el reconocimiento de sus características y el fortalecimiento de la identidad (Giménez, 2009), lo que puede redundar en una contribución mayor a la relación positiva en la ciudad. Los Jóvenes indígenas universitarios relatan su experiencia de sentir discriminación activa (en la escuela o la calle) y pasiva (a través de la falta de oportunidades o programas específicos) dada su condición de joven, 
indígena y estudiante universitario en una ciudad poco proclive a reconocerlo (Negrín da Silva, 2010). En este sentido, compartir atributos idiosincráticos o relacionales, así como experiencias de vida (Giménez, 2009) apoya el reconocimiento identitario.

En cuanto al recuento de la experiencia, cada uno fue capaz de reflexionar, participar y generar compromisos y propuestas para reforzar su cultura y darse cuenta de la situación que está viviendo. Se inconforma con la situación actual, pero intenta, como sujeto colectivo, trascender la incomodidad para ir hacia la propuesta. Al mismo tiempo, reconoce que vivir en la ciudad tiene aspectos negativos y positivos de los que se pueden servir en el trabajo colectivo.

El balance de estos encuentros y reflexiones es positivo. Los Jóvenes indígenas universitarios han reconocido esta experiencia como valiosa, pero se dan cuenta que se trata de un proceso largo que apenas inicia. La capacidad de poner en común las vivencias y problemas, así como propuestas de acciones concretas los motivan a seguir adelante $y$, de alguna manera, fortalecen la identidad múltiple de joven + indígena + universitario. Los siguientes pasos dependen de la capacidad del colectivo de asumirse como tal y deben darse con parsimonia, reconociendo que su proceso requiere ser consciente, crítico y autocrítico ante la realidad en que se vive.

Lo presentado en el cuerpo de este trabajo marca la necesidad de los Jóvenes indígenas universitarios de hablar sobre los procesos que viven y que ningún otro espacio garantiza; además, permite conocer la situación que se da en un contexto hostil a la diversidad cultural, pues es en ese espacio donde tienen que vivir, es decir, ya forman parte de ese contexto, ya que por eso surge la inquietud de conocer más allá de lo que comparte un grupo más amplio sobre los temas aquí tocados. Esta experiencia impulsó al grupo a generar espacios para visibilizar la presencia indígena en la ciudad a través de diálogos con instituciones, indígenas y no indígenas, que años más tarde, en 2017, resurgieron como Jóvenes Indígenas Urbanos, cambiando lo universitario a urbano, para marcar otro proceso del colectivo, paralelo a la discusión de la identidad indígena en un contexto urbano, más allá de haber conservado los integrantes del equipo base y promotor, se suman 
más, ahora dando mayor énfasis a las acciones desde las perspectiva intercultural y la cosmovisión de los pueblos originarios en el contexto urbano.

\section{REFERENCIAS BIBLIOGRÁFICAS}

Acosta, L. (2005). Guía práctica para la sistematización de proyectos y programas de cooperación técnica. Santiago de Chile: Oficina Regional de la FAO para América Latina. Recuperado de http://www.fao.org/3/a-ah474s.pdf

Asociación Nacional de Universidades e Instituciones de Educación Superior (ANUIES). (2007). Matrícula PAEIIES. Recuperado de http://paeiies.anuies.mx/public/index. php? pagina $=$ cobertura.html

Ávila, I. (2010). El problema del indio. Indigenismo en México, 1934-1940. (Tesis de licenciatura). Universidad Nacional Autónoma de México, México.

Badillo, J., Casillas, M. y Ortiz, V. (2008). Politicas de atención a los grupos indigenas en la educación superior mexicana: el caso de la Universidad Veracruzana. Recuperado de http:// udlap.redalyc.org/articulo.oa?id=55261004

Borja, J. y Castells, M. (2000). Local y global. La gestión de las ciudades en la era de la información. México: Taurus.

Casillas, M. L. y Santini, L. (2006). Universidad intercultural. Modelo educativo. México: SEP, Coordinación General de Educación Intercultural y Bilingüe. Serie Documentos

Castells, M. (1998). La era de la información. Economía, sociedad y cultura. Vol. 2 El poder de la identidad. Madrid: Alianza.

De la Peńa, G. (2005). Los nuevos intermediarios étnicos, el movimiento indígena y la sociedad civil: dos estudios de caso en el occidente mexicano. En Sociedad Civil, Espacio Público y Democracia en México, (pp. 1-43). México: Universidad Veracruzana.

De la Peña, G. (2010). ¿Una nueva categoría analítica? Los indígenas urbanos en la antropología mexicana: La antropología y la conciencia nacional mexicana. En El Colegio de Jalisco, (pp. 1-25). México: Centro de Investigaciones y Estudios Superiores en Antropología Social (CIESAS). 
Dirección General de Educación Indígena (DGEIB). (S/F). Breve Historia. Recuperado de http://basica.sep.gob.mx/dgei/ pdf/inicio/conocenos/historiaDGEI.pdf

Domínguez, R. F. (2011). Zoques en la ciudad de Guadalajara: La reproducción de una identidad étnica dispersa. (Tesis de Maestría). CIESAS Occidente, Jalisco, México.

Domínguez, F. (2013). La comunidad trasgredida: los zoques en Guadalajara. Guadalajara, Jalisco: Unidad de apoyo a las Comunidades Indígenas de la Universidad de Guadalajara.

Durín, S. (2007). ¿Una educación indígena intercultural para la ciudad? El departamento de educación indígena en Nuevo León. El colegio de la Frontera Norte: Tijuana, (19)38, 63-91.

Emiro, E. (2005). Investigación Acción. Metodología Transformadora. Santa Rita, Zulia: Universidad Nacional Experimental Rafael María Baralt.

226 Giménez, G. (2009). Identidades Sociales, México: Consejo Nacional para la Cultura y las Artes.

Gómez, N. D. (2006). La experiencia de la diversidad cultural en una institución de educación superior; un estudio exploratorio de algunas de las representaciones sociales de actores universitarios. (Tesis de maestría). ITESO, Jalisco, México.

Hamelink, C.J. (1989). The Relationship between Cultural Identity and Modes of Communication. En J. A. Anderson (Ed.) Communication Yearbook/12 (pp. 417-426). Londres: Sage.

Instituto Nacional de Estadística y Geografía (INEGI). (2012). Estadísticas a propósito del día internacional de los pueblos indígenas. Datos de Jalisco. Guadalajara, Jalisco: INEGI.

Instituto Nacional de Estadística y Geografía (INEGI). (2010). Migración. Recuperado de http://cuentame.inegi.org.mx/ poblacion/migracion.aspx?tema $=\mathrm{b}$

Instituto Nacional de Estadística y Geografía (INEGI). (2012). Estadisticas a propósito del Día Internacional de los Pueblos Indigenas. Datos de Jalisco. Recuperado de http://www. inegi.org. $\mathrm{mx} /$ inegi/default.aspx?c=274 
JÓVENES INDÍGENAS UNIVERSITARIOS EN LA ZONA METROPOLITANA DE GUADALAJARA

Instituto Nacional de Estadística y Geografía (INEGI). (2015). Panorama Sociodemográfico de Jalisco. Recuperado de http://internet.contenidos.inegi.org.mx/contenidos/productos/prod_serv/contenidos/espanol/bvinegi/productos/nueva_estruc/inter_censal/panorama/702825082239. pdf

Jara, O. H. (1994). ¿Cómo sistematizar? Una propuesta en cinco tiempos. Presentación, Centro de Estudios y Publicaciones (CEP). San José, Costa Rica: ALFORJA.

JIU (2014). El Informe JIU 2013-2014. Documento interno no publicado.

Korsbaek, L., Sámano-Rentería, M. Á. (2007). El indigenismo en México: antecedentes y actualidad. Ra Ximhai, 3(1), 195-224. Recuperado de http://www.redalyc.org/ pdf/461/46130109.pdf

Martínez, R. y Rojas, A. (2006). Indígenas urbanos en Guadalajara: etnicidad y escuela en niños y jóvenes otomíes, mixtecos y purépechas. En P. Yanes, V. Molina y Ó. González (Coords.). El triple desafío. Derechos, instituciones y politicas para la ciudad pluricultural, México: Universidad de la ciudad de México.

Negrín da Silva, D. (2011). Guadalajara de las Indias: Quinientos años de construcción étnica en la Perla Tapatía. Lucero Journal: A Journal of Iberian and Latinamerican Studies. Recuperado de http://escholarship.org/uc/item/3f79n63t

Rahman, M. A. y Fals-Borda, O. (1989). La situación actual y las perspectivas de la IAP en el mundo. Análisis Político, 5, 14-20.

Reguillo R. (2003). Las culturas juveniles: un campo de estudio; breve agenda para la discusión. Revista Brasileira de Educação, 23, 103-118.

Saraví, G. (2010). Presentación: Juventud indígena en México: tensiones emergentes para el análisis y las políticas públicas. AQUí estamos Revista de Exbecarios Indigenas del IFP-México, 13, 5-10.

Schmelkes, S. (2011). Las Universidades Interculturales en México: ¿Una contribución a la equidad en educación superior? 
Recuperado de http://www.colombiaaprende.edu.co/ $\mathrm{html} /$ mediateca/1607/articles-174704_archivo.pdf

Souto K. S. (2007). Juventud, Teoría e Historia: La formación de un sujeto social y de un objeto de análisis. Historia Actual Online, 13, 171-192.

Valadez, I., González, J., Vargas, V., Ramírez, H., Alfaro, N., Fausto, J., Montes, R., Orozco, M., Luevanos, A., Laureano, A. y Rodríguez, J. (2013). La educación para la salud, en procesos de intervención participativa. México: Secretaria de Salud.

Urteaga, M. (2017). México: “Jóvenes indígenas". Flujos étnicos contemporáneos y giros epistémicos. En Da Costa y Rangel (Coords.) Juventudes Indigenas, estudos interdiciplinare, saberes interculturais conexöes entre Brasile México. Riode Janeiro: e-papers. 